\title{
Dielectric Barrier Discharge (DBD) Plasma Actuators Thrust - Measurement Methodology Incorporating New Anti-Thrust Hypothesis
}

\author{
David E. Ashpis ${ }^{1}$ \\ National Aeronautics and Space Administration, Glenn Research Center, Cleveland, Ohio, 44135 \\ and \\ Matthew C. Laun ${ }^{2}$ \\ Sierra Lobo Inc., Glenn Research Center, Cleveland, Ohio, 44135
}

\begin{abstract}
We discuss thrust measurements of Dielectric Barrier Discharge (DBD) plasma actuators devices used for aerodynamic active flow control. After a review of our experience with conventional thrust measurement and significant non-repeatability of the results, we devised a suspended actuator test setup, and now present a methodology of thrust measurements with decreased uncertainty. The methodology consists of frequency scans at constant voltages. The procedure consists of increasing the frequency in a step-wise fashion from several $\mathrm{Hz}$ to the maximum frequency of several $\mathrm{kHz}$, followed by frequency decrease back down to the start frequency of several $\mathrm{Hz}$. This sequence is performed first at the highest voltage of interest, then repeated at lower voltages. The data in the descending frequency direction is more consistent and selected for reporting. Sample results show strong dependence of thrust on humidity which also affects the consistency and fluctuations of the measurements. We also observed negative values of thrust or "anti-thrust", at low frequencies between $4 \mathrm{~Hz}$ and up to $64 \mathrm{~Hz}$. The anti-thrust is proportional to the meansquared voltage and is frequency independent. Departures from the parabolic anti-thrust curve are correlated with appearance of visible plasma discharges. We propose the antithrust hypothesis. It states that the measured thrust is a sum of plasma thrust and antithrust, and assumes that the anti-thrust exists at all frequencies and voltages. The anti-thrust depends on actuator geometry and materials and on the test installation. It enables the separation of the plasma thrust from the measured total thrust. This approach enables more meaningful comparisons between actuators at different installations and laboratories. The dependence on test installation was validated by surrounding the actuator with a large diameter, grounded, metal sleeve.
\end{abstract}

\section{Nomenclature}

$f \quad=$ frequency

$k \quad=$ anti-thrust coefficient

$V \quad=$ mean voltage

Actuator geometry parameters see Appendix A

Acronyms

AC Alternating Current

DC Direct Current

RH Relative Humidity

Subscripts

p-p, pp peak to peak

\footnotetext{
${ }^{1}$ Research Aerospace Engineer, Glenn Research Center, 21000 Brookpark Road, Associate Fellow AIAA.

${ }^{2}$ Electrical Engineer, Glenn Research Center, 21000 Brookpark Road, Nonmember.
} 


\section{Introduction}

A DIELECTRIC Barrier Discharge (DBD) actuator is a device that consists of a pair of electrodes separated by a dielectric. One electrode is exposed to the flow and the other is covered. Alternating Current (AC) voltage in the kilovolt $(\mathrm{kV})$ range is applied to the electrodes and creates weakly ionized surface discharge plasma on the exposed electrode edge towards the buried electrode direction (Fig.1). There are usually two modes of operation. Conventional DBD operation involves application of an Alternating Current (AC) voltage waveform in the kilohertz frequency range, with or without modulation or pulsing. In this mode, referred to as "AC DBD", heat generation is insignificant and the actuator generates momentum in the form of a wall-jet parallel to the surface. The wall-jet momentum coupling with the external flow is the foundation for active flow control. The other mode of DBD operation involves applying voltage consisting of ultra-short, repetitive pulses. The pulses are usually several nanoseconds wide and the repetition rate ranges from a few hundred

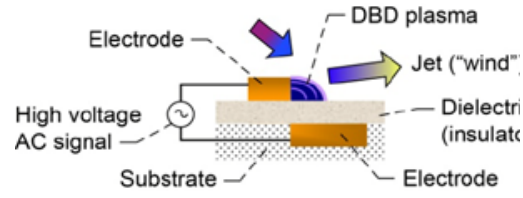

Figure 1. Schematic of a DBD Plasma Actuator hertz to a few hundred kilohertz. In this mode of operation, generally referred to as "NS-DBD," the generated momentum is negligible but there is a fast, localized heating of the gas that creates pressure waves or even shock waves. The repetition generates pressure oscillations that are used for active flow control. For the momentum-based DBD actuator, see review articles by Moreau ${ }^{1}$ and Corke et $a l^{2,3,4}$. For the ultra-short pulsed fast heating-based DBD actuator, see the paper by Roupassov et al $^{5}$.

This project is limited to the conventional AC-DBD, momentum-generating DBD actuator with AC operation (kilohertz-range applied voltage frequency). The interest is to characterize the aerodynamic and electrical performance of the actuator. Generally, it is performed without external flow. This paper is focused on the actuator thrust, addresses related issues and proposes measurement and data interpretation approaches.

\section{The Thrust of the DBD Actuator}

The thrust of the DBD plasma actuator is a good metric for its aerodynamic performance. The reason is that in active flow control application the main interest is in the momentum injected into the flow by the actuator. Pneumatic actuators use a momentum coefficient as performance and characterization parameter (e.g. Glezer and Amitay ${ }^{6}$ ).

The momentum can be calculated from flow velocity profiles measurements, or from direct thrust measurement. From a control volume consideration shown in Fig. 2, the thrust is approximately equal to the net momentum generated by the actuator ${ }^{7,8}$. The difference is the shear force on the surface. This is for the streamwise, or parallel to the surface, direction. There is also a component of the force in the surface-normal direction not considered here.

An attractive reason to use thrust is simplicity. The thrust can be measured by a balance or a load cell. Because load cells with the required resolution, range and tare weight capacity are hard to find or non-existent, an analytical balance is useful for this suppose. It is desirable to use one with milligram (mg) resolution. When the actuator is placed on the balance with the jet facing up as shown in Fig. 3, the balance reading is the sum of the forces generated by the plasma and the shear forces on the surface. If the shear forces are sufficiently small to be neglected, then the thrust will be close to the momentum ${ }^{7}$.

\section{A. Shear forces and their relation to thrust}

The magnitude of the shear forces is in question. Experiments by Durscher and Roy

${ }^{9}$ showed that the thrust measured was dependent on the distance from the edge of the exposed electrode to the edge of the actuator. The conclusion would be that the shear forces are not negligible. Experiments by Opaits et $\mathrm{al}^{10}$ showed that the generated walljet is Glauert's self-similar wall-jet ${ }^{11}$. Opaits has not calculated the wall shear forces. We have used his data and calculated them (to be reported separately), and found that the calculated shear force is negligible. However these calculated shear forces apply only to the self-similar wall-jet domain. The region near the electrode edge can be considered as the near-field source of the wall-jet, where the similarity is not valid. Calculation of the shear stresses on the surface in the near-field is a complex task that involves solving plasma chemistry equations. There is no reason to assume that the

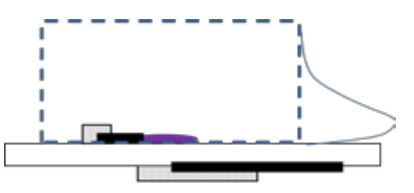

Figure 2. Control volume for thrust evaluation

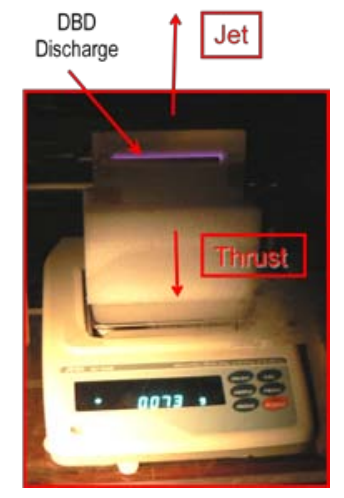

Figure 3. Setup of an actuator on an analytic balance 
shear is negligible. However, the extent of that region is small (less than $10 \%$ of the total length measured by Opaits et $\mathrm{al}^{10}$ ), therefore the shear stress will act on a very small portion of the actuator surface area. It seems reasonable to assume that the resulting shear forces will be small. Assessment of this conclusion is in progress and will be reported separately.

Regardless of the accuracy of the thrust as representing the momentum, it can be considered a good aerodynamic performance metric of DBD actuator on its own merit.

\section{B. Problems with direct wall-jet velocity measurement}

Thrust measurements do not provide information on the wall-jet velocity profile. The other class of methods involve direct measurements of the wall- jet profiles, from which the momentum can be calculated. Pitot and hotwire probes require flow traverses. Inserting probes near plasma and electric fields causes various problems. Metallic probes introduce an equipotential body into the flow and induced charges that change the electrical field and hence the velocity field in its vicinity. Arcing to the dielectric and the electrodes can occur, also affecting the flow (Fig. 4). We have found that metallic probes behave as grounded probes even when they were not grounded and were isolated from the test setup by Tygon $^{\circledR}$ tubing and dielectric fixtures. Therefore dielectric materials, e.g. glass, are used. They are believed to be suitable and widely used, but they also can accumulate charges on their surface that will alter the electrical field in the vicinity and change the local velocity. The difference compared to metals is that is that these charges are static and not moving, and the surface is not equipotential as in metals.

Non-intrusive methods include Particle Image Velocimetry (PIV) and Laser Doppler Velocimetry (LDV). They require seeding and questions arise as to the effect of the seeding. The seeding material can become electrically charged and the electrostatic forces prevent it from following streamlines. Several researchers have compared PIV and LDV methods with Pitot probe and hot wire measurements and found close results, indicating that seeding charging is not an issue. However Boucinha et $\mathrm{al}^{12}$ have measured the wall-jet profile with a glass Pitot tube under different seeding materials (Fig. 5), and there are marked differences. It indicates that the seeding material participates in the plasma chemistry process or changes the surface chemistry or surface charging by adhering to it, with the end result affecting the performance of the actuator.

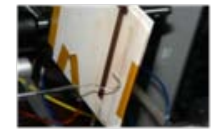

(a)

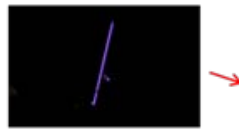

(b)

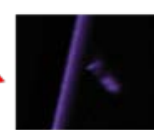

(c)

Figure 4. Metal Pitot probe arching to an Alumina dielectric. (b) is same image as (a) but in the dark. (c) is enlargement showing arcing.

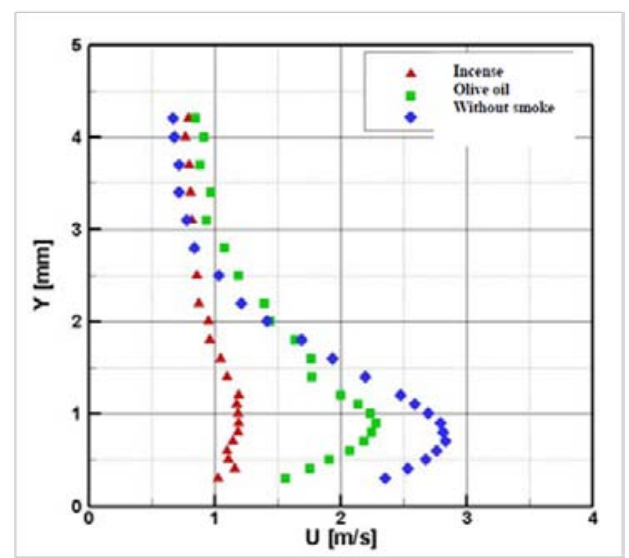

Figure 5. Effect of seeding on the wall-jet generated by the actuator. Reprinted with permission from Boucinha et al $^{12}$, AIAA 2008-4210, Copyrighted to the authors.

In light of these difficulties it appears that thrust measurement is an attractive and simple method to characterize the performance of DBD actuators.

\section{Application of thrust data}

Our ultimate goal is to perform a comprehensive characterization of the aerodynamic and electrical performance of DBD plasma actuators. The tests would be performed without external flow in room conditions and in vacuum and pressure chambers. The objective is to perform parametric studies to create a data base of thrust vs. electric power consumption of DBD actuators for the canonical configuration of an actuator constructed of 2D offset electrodes. The parameters would be the voltage, frequency, waveform, and duty cycle of the applied voltage as well as the actuator geometry, dielectric properties, and ambient conditions such as humidity, pressure and temperature. An example of a performance map is shown in Fig. 6 for sinusoidal wave form. These types of multi-dimensional maps can be used for selection of actuators, establishing requirements for power supplies, and optimization of the actuation system. The power consumption measurements were addressed in Ashpis et al ${ }^{13}$, and when thrust measurements were attempted, we encountered difficulties in obtaining consistent results. 


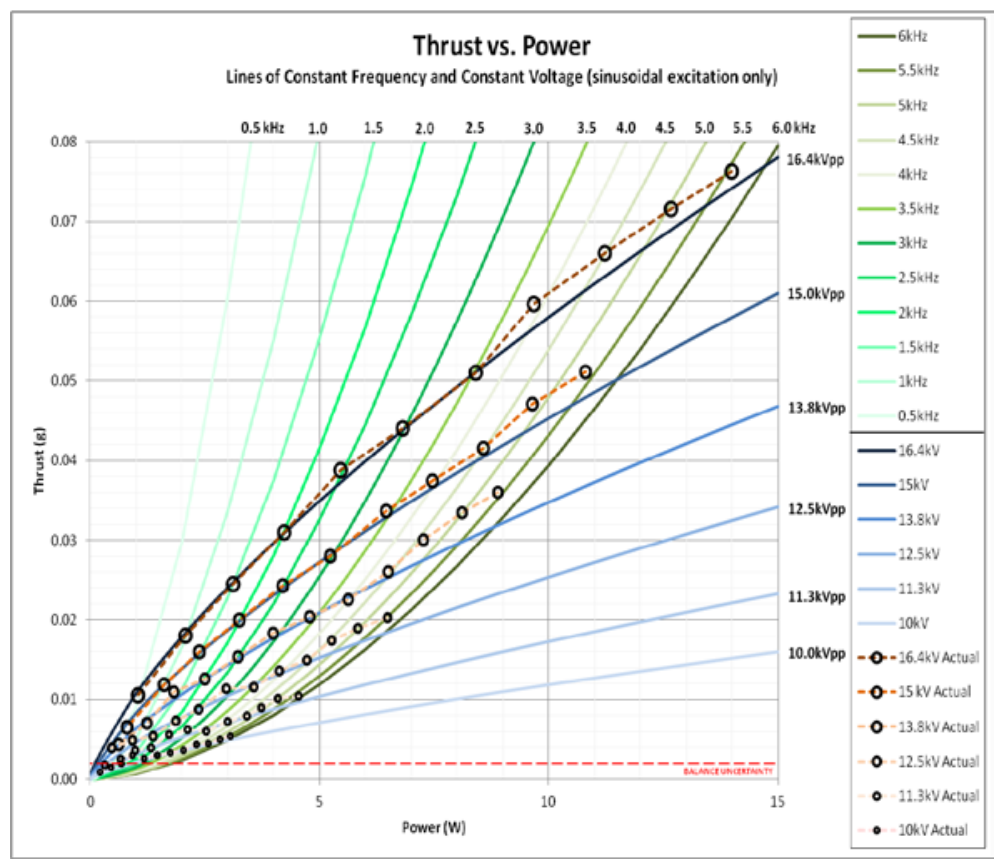

Figure 6. DBD actuator sample performance map. The curves are to assist reading the data, not necessarily valid beyond the measured data.

\section{Consistency of thrust measurements.}

We initially performed thrust measurements with various actuators held with acrylic fixtures in an acrylic enclosure. The balances used were AND (A\&D Company, Limited, Japan) Models GF-300 and GF-1000. The readings were recorded using the balance's serial port and a LabView ${ }^{\mathrm{TM}}$ program. The power was provided with thin copper wires (24 to 32 AWG), whose static deflection did not affect the balance reading. It was tested by placing weights in presence of an unpowered actuator, and comparing the balance reading to the applied weight. This balance displays good immunity to Electromagnetic Interference (EMI), but was placed inside a copper Faraday cage as extra measure. A copper ground plane was used for appropriate grounding.

In the course of the tests, performed with acrylic and alumina actuators, we observed several problems. We found large degrees of non-repeatability, fluctuations, and drift in time. There was strong dependence on actuator conditioning profile ("burn-in"). This was also observed by others (e.g. Hoskinson et al ${ }^{7}$ ). There was dependence on the manner of applying the voltage. For example, if the voltage was increased at fixed frequency, the

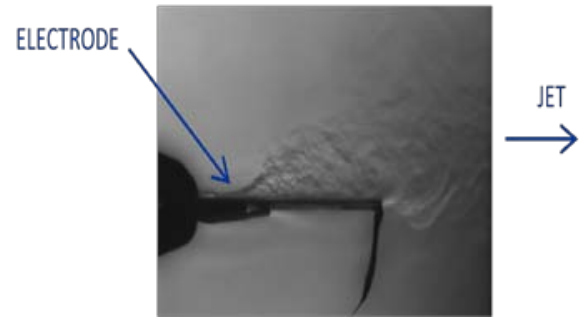

Figure 7. Focusing Schlieren image of an alumina actuator.

Density gradients are caused by surface heating. The image generated with MetroLaser Inc. Strioscope dual grid projection focusing Schlieren system. readings were different if the voltage was increased from one measuring point to the next, or if it was brought to zero between the measurement points. When the high-voltage lead-wire was charged, it was performing oscillatory motions, indicating dynamic forces caused by charges, ionic wind, corona discharge, and elasticity of the wire; significant amplitudes were observed at some setups. 
There were indications that the measured thrust was affected by several phenomena. There was apparent interaction of the actuator with the enclosure, nearby objects, and the ground plane. Charges appeared on the outside surface of the acrylic enclosure. The actuator dielectric itself exhibited charges that appeared to be due to remnant polarization, ferroelectric activation, and possible paraelectric behavior.

Thrust fluctuations could originate from flow recirculation inside the enclosure and from strong unsteady walljet wake fluctuations observed at its departure from the plate edge. The latter was visualized in the Schlieren photograph in Fig. 7.

Finally, there was strong dependence on humidity. The effect is well known in the atmospheric pressure plasma research community, see for example, Koo et $\mathrm{al}^{14}$, attributing the effect on changes in the plasma chemistry caused by generation of $\mathrm{OH}$ radicals via interaction of electrons and the water molecules affecting the plasma chemistry. However when reviewing publications reporting experiments on DBD actuators performance, we found that humidity conditions are rarely reported.

Problems with thrust measurements were also observed by others. They were mostly associated with the initial conditioning or "burn-in" and various strategies were employed. The reader is referred to a more recent reference, Durscher. \& Roy ${ }^{9}$. Unlike our experience, they have not observed significant thrust drift in their tests, but report several open problems and areas that need further investigation associated with actuators' thrust measurements.

External circuit parameters are also a factor in actuator performance ${ }^{15}$. In the work reported here we have not made any changes affecting the external circuit impedance. The same power supply, supply cabling, and feedwires are used. The only impedance change between different tests is the impedance of the actuator test article and impedance change caused by frequency dependence.

Based on our experience with our thrust measurements attempts and considering the problems described above, we developed a new test setup and a new methodology for thrust measurement. Our methodology also led us to the proposed anti-thrust hypothesis used to separate the relevant plasma thrust from the total measured thrust. These approaches will be described below.

\section{Test setup}

We have developed a test setup to counter some of the problems associated with our testing in an enclosure. The new test setup is shown in Fig. 8. The AND analytic balance we used (A\&D ${ }^{\circledR}$ Company, Limited, Japan, Model AND GX-1000) has linear accuracy of $\pm 3 \mathrm{mg}$ and repeatability of $1 \mathrm{mg}$. It is equipped with an underhook that enables it to measure hanging loads. The balance was installed on a small aluminum platform attached to the laboratory ceiling. The balance was thermally insulated with an enclosure made from polystyrene foam sheets to minimize thermal drift. The air temperature in the enclosure was monitored with a thermocouple. The actuator test article was suspended with thin nylon monofilaments (fishing line) attached to a metal frame that was hung on the balance's hook. The test article was installed as far as practical from nearby objects. The floor underneath the test article consisted of grounded metal plates. The surrounding objects included metal cabinets, workbenches, metal and acrylic structures, and cement walls and floors with embedded steel reinforcing. The balance was installed about $3.5 \mathrm{~m}$ above the actuator, and testing revealed there was no detectable EMI interference by the balance due to the actuator. The nearest distance to adjacent objects was $1.2 \mathrm{~m}$. Typically the distances were in the range of 1.5 to $2.0 \mathrm{~m}$. The actuator was suspended about $1.2 \mathrm{~m}$ above the floor. After gaining some experience, we were able to minimize movements of the suspended test article that would affect the balance reading.

We used a Trek Inc. high voltage amplifier Model PD06035. Its maximum slew rate is $725 \mathrm{~V} / \mu$ s (at no load, $10 \%$ to $90 \%$ typical) and the DC gain is $3000 \mathrm{~V} / \mathrm{V}$. The effective slew rate was reduced to $245 \mathrm{~V} / \mu \mathrm{s}$ after the electrical actuator load was applied. The range of its working

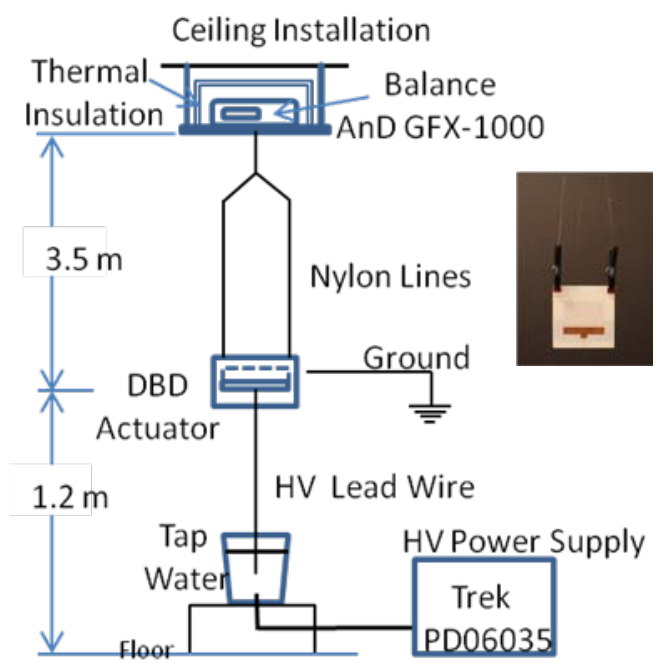

Figure 8. Suspended actuator test setup schematic (not to scale)

frequencies starts at DC. The combination of its frequency and voltage output range is limited by the slew rate (and the load impedance). The sinusoidal input waveform was supplied with a synthesized signal generator, Stanford 
Research Systems (SRS) Model DS345m. The Trek is equipped with a variable-intensity indicator to warn of output waveform distortion. We also simultaneously used an oscilloscope to detect output voltage distortion.

We used the factory-supplied output cable, Trek part number 43466B, with a total length of approximately $2 \mathrm{~m}$. A section of the cable, approximately $1.5 \mathrm{~m}$ long, was routed through 1 inch I.D. acrylic and PTFE tubes for extra insulation above the metal floor. This arrangement was kept constant at the various tests so as not to vary the capacitance between the cable and the surroundings.

The high voltage was fed to the powered actuator electrode through a conductive-liquid, force-decoupling interface arrangement as follows. The high voltage 28 AWG copper feedwire lead was suspended vertically with a metal counterweight into a styrene cup containing tap water that submerged the counterweight completely below the surface. The high voltage was fed from the output cable of the power supply into the water via a stainless steel needle that pierced the bottom of cup. Sufficiently ionized tap water was selected due to its conductivity and allows charging of the actuator electrode with minimal impedance. Dynamic forces caused by the lead wire, observed before with different setups, were minimized below detectable levels. The ground 28 AWG copper feedwire lead was connected to the covered electrode via a thinner 40 AWG copper wire suspended in an approximate catenary shape to minimize forces.

The high voltage was measured with the Trek's built-in voltage monitor (3000:1 ratio). It is based on a high performance voltage divider. It adequately represents the AC voltage on the electrodes within the moderate frequency ranges used. The built-in current monitor was not used for data as our tests showed unsatisfactory highfrequency dynamic response. We have not recorded the current as it was not within the objectives of this project.

We used a Nikon digital camera Model D300S to take still images of the discharges. Camera setting of the images shown herein were F2.8, ISO 200, and exposures of 30 seconds or as noted. Darkened room was required.

The balance readings were recorded using a LabView ${ }^{\mathrm{TM}}$ application. The balance provides continuouslyaveraged load measurements 10 times per second. Testing performed on the balance revealed that this averaging occurs for time-varying loads at frequencies above $2 \mathrm{~Hz}$. Alternatively, time-accurate readings can be acquired for loads that vary below $0.5 \mathrm{~Hz}$. The accuracy of the AND balance is accomplished via a servo loop activating an electromagnetic coil that maintains the deflection of the internal beam at zero. The electrical current to the coil is nearly linear with the load. This construction has a particular advantage relevant to our tests, as the static forces of the lead wires are null, because there is no steady state deflection that will cause stress forces in the lead wires.

The actuators we used in the study reported here were made of $6.3 \mathrm{~mm}$ (0.25 in) High-Density Polyethylene (HDPE) dielectric. The dielectric properties (see Appendix A) are close to those of PTFE, Dielectric Constant $\varepsilon=2.3$, making it a low capacitance class of actuators that were shown by others to allow application of high voltage to achieve high levels of thrust. We found that this material does not exhibit sudden pin-hole type burn-through that we encountered while using PTFE, PEEK, and other polymers. The HDPE exhibited excellent durability over long periods of time. The dimensions and other construction details of the actuators used are listed in Appendix A.

\section{Thrust measurement methodology}

Usually, there is a need to perform a large number of thrust measurements within a test matrix indexed by frequency and voltage. We need to minimize the testing time while considering the issues described above and also to take steps to obtain consistent results. To acquire the two-dimensional thrust data matrix, data must be acquired at constant voltage or at constant frequency. Since we have more experience with the magnitude of thrust errors that are dependent upon voltage than those due to frequency, we favor acquiring data by maintaining constant voltage. Thrust data must be averaged over time at each pair of voltage and frequency.

We are also trying to avoid performing numerous repetitions that would lead to comprehensive statistical analyses. Repetitive tests may take long periods of time, several hours or several days, and changes may occur in the actuator, due to changes in ambient conditions (humidity, temperature), chemical reactions with the surface, erosion of the electrodes and the dielectric, dielectric heating, changes in the adhesives used, moisture absorption, and potential net charge non-equilibrium. If tests are performed in a closed chamber, changes of the surrounding gas composition can occur by accumulation of plasma-generated Ozone and other species. There are also questions as to what is the appropriate period of data recording time used to calculate the average thrust for each point in the voltage-frequency matrix.

We use a "burn-in" process before data acquisition to condition the actuator to minimize potential long-term voltage-dependent thrust error. Our process is to expose the dielectric and surfaces to the maximum absolute voltage as well as to the maximum voltage slew rate with a sufficient dwell time to instigate the initial change. This change 
occurs relatively quickly (typically 3-10 minutes). Once changed, the actuator zero-thrust reference remains stable for a longer period of time (2-3 hours) that facilitates the long-term acquisition of the data matrix.

Based on our experience and trials with different actuators, we developed a methodology of acquiring actuator thrust data using discrete frequency sweeps at constant voltages and recording average readings of the balance, as follows.

First, the ranges of voltage and frequency of interest are determined. They are limited by the performance specifications of the power supply, mainly governed by its slew rate, the load impedance, and the breakdown voltage of the actuator dielectric. Next, the voltage is set to the highest voltage in the range, $(48 \mathrm{kVp}-\mathrm{p}$ in this example). The frequency is set to a low number $(4 \mathrm{~Hz})$ and then increased to the maximum frequency in the range $(2048 \mathrm{~Hz})$ in discrete frequency steps, each lasting 60 seconds. The discrete frequencies are distributed in a logarithmic fashion with octave spacing. That is, each step is double the frequency of the prior step.

After the maximum frequency is reached, the process continues by decreasing the frequency down from the maximum frequency $(2048 \mathrm{~Hz})$ with the same discrete, 60 second steps, halving the frequency at each step down to the lowest frequency $(4 \mathrm{~Hz})$. That completes one fixed-voltage data series, a row in the matrix. The process is repeated at the next lower fixed-voltage (42 kVp-p). It is followed by repetitions at the other fixed-voltages in the decreasing direction until the matrix is filled. We refer to this process as "Frequency Sweeps".

An example of Frequency Sweep raw data is shown in Fig. 9 showing thrust versus time for each fixed voltage. The actuator used is actuator HDPE \#3 (Appendix A). The Relative Humidity (RH) was $50 \%$ and the correlated dew point was $57^{\circ} \mathrm{F}$ (averaged between four tests). The thrust data was collected after the actuator was inactive and unenergized for 4 consecutive days. The initial "burn-in" procedure was intentionally skipped in order to exhibit the need for one.

Examining Fig. 9, the first data row of the matrix, acquired at a constant $48 \mathrm{kVp}-\mathrm{p}$, clearly shows large asymmetry and hysteresis. Fig. 13 reinforces this observation. This distortion and non-repeatability is largely attributable to the lack of a prior "burn-in". Further examination of Fig. 9 reveals that there is still mild asymmetry between the ascending and the descending halves of the other frequency sweeps even after the first sweep at 48 kVp-p provided a partial "burn-in". The thrust levels acquired at the same frequency and voltage differ instead of matching. The levels appear to depend on the frequency change direction. Further, the small time-scale thrust fluctuations are different. The $48 \mathrm{kVp}-\mathrm{p}$ curve is distinguished by stronger initial spikes and fluctuations as compared to those at subsequently lower constant-voltages. The behavior of the thrust fluctuations in the descending frequency sweeps is generally smoother and more consistent than in the ascending sweeps. Therefore, the descending data is recommended for further processing (shown in subsequent section).

We have accompanied our measurements with still images taken with a digital camera (10 second exposures) as shown in Fig. 10. Taken at the same frequency and voltage, they show differences in the discharge structure that depend on the direction of the frequency step change. Also, there are dark areas within the plasma region on both directions of the frequency sweep that we cannot explain. On the ascending frequency side, bright localized filaments are observed, however, they are absent from the descending side. It is possible that they disappeared after the partial "burn-in" process (during prior frequency steps at $48 \mathrm{kVp}-\mathrm{p}$ ) that conditioned the actuator surface and electrodes.

A different case is shown in Fig. 11. The test actuator is the same HDPE \#3 as the former, but with additional insulation comprised of several layers of Kapton ${ }^{\circledR}$ tape $^{3}$ and corona dope ${ }^{4}$ used as filler on the side and back (upstream) edges of the exposed electrode. The added insulation was intended to suppress visible "parasitic" corona discharge at electrode corners with very small radius. It was desired to minimize potential thrust activity from localized electrode sites so as to focus on the thrust from the linear actuator edge discharge alone. The ambient humidity was much drier compared to the previous case: RH 18\%, Dew Point $33^{\circ} \mathrm{F}$. There are marked differences in the results. The steps are more uniform, fluctuation levels lower, and there is more symmetry between the ascending and descending parts. Images shown in Fig. 12 show a uniform discharge without observable difference between the ascending and the descending frequency parts. We notice that the dark areas within the plasma region observed in Fig. 10 disappeared. We attribute the differences mainly to the lower humidity level, this trend was confirmed by additional tests not reported here. The additional insulation contributes to reduce fluctuations and increase smoothness of the frequency-dependency curves.

\footnotetext{
${ }^{3} 3 \mathrm{M}^{\circledR}$ No. 5413, $0.08 \mathrm{~mm}$ (3 mil) thick

${ }^{4}$ MG Chemicals ${ }^{\circledR}$ Inc. Cat. No. 4226-1L
} 


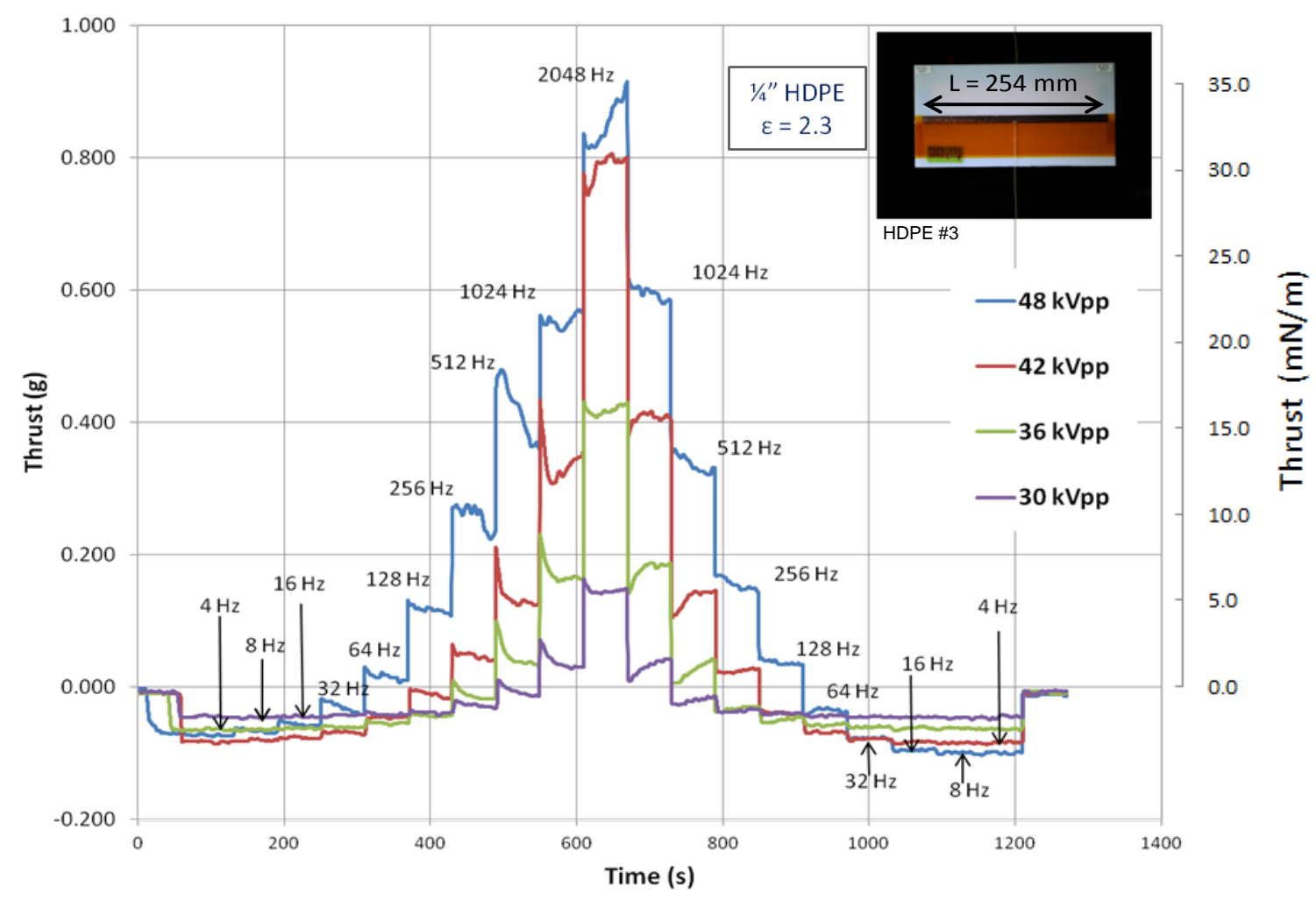

Figure 9. Frequency sweeps at constant voltages. RH 50\%, Dew Point $57^{\circ} \mathrm{F}$. Sequence started at $48 \mathrm{kV}$ after 4 days rest. $1 \mathrm{~g}$ equals $38.6 \mathrm{mN} / \mathrm{m}$.

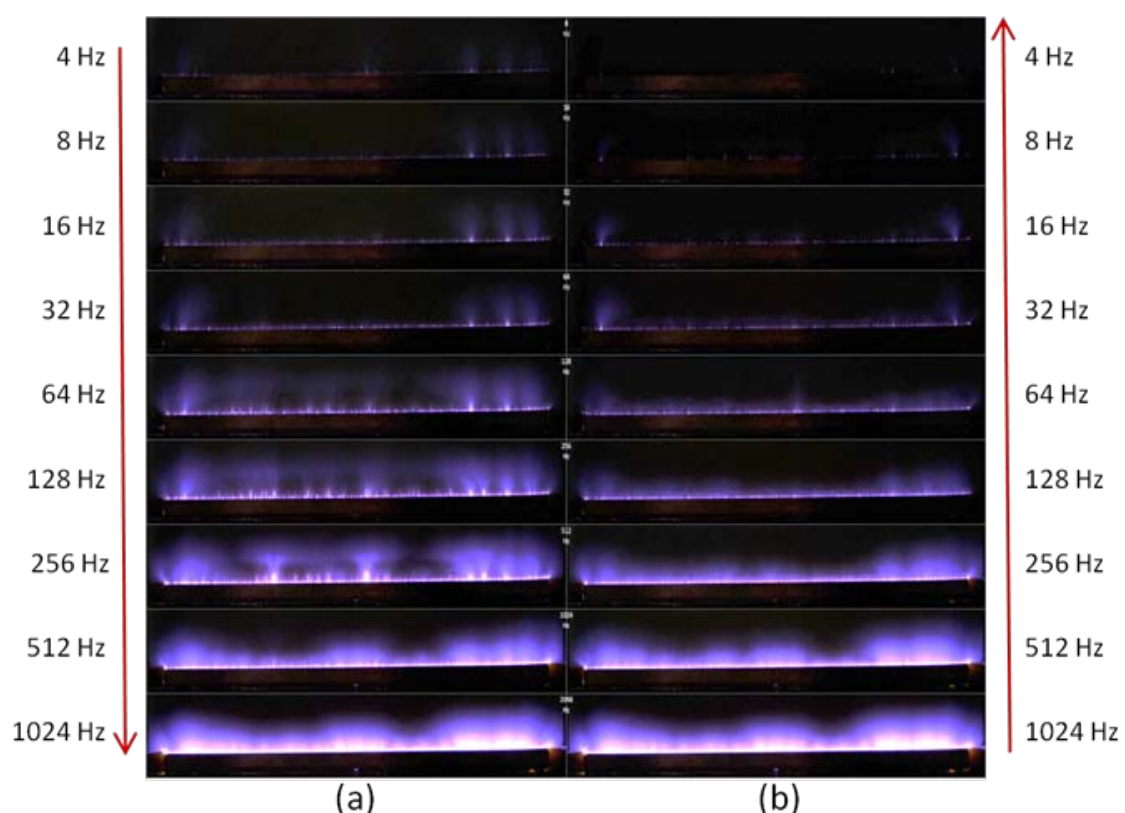

Figure 10. Images of $\mathbf{4 8} \mathbf{k V p}-\mathrm{p}$ frequency sweep discharges corresponding to Fig. 9. 


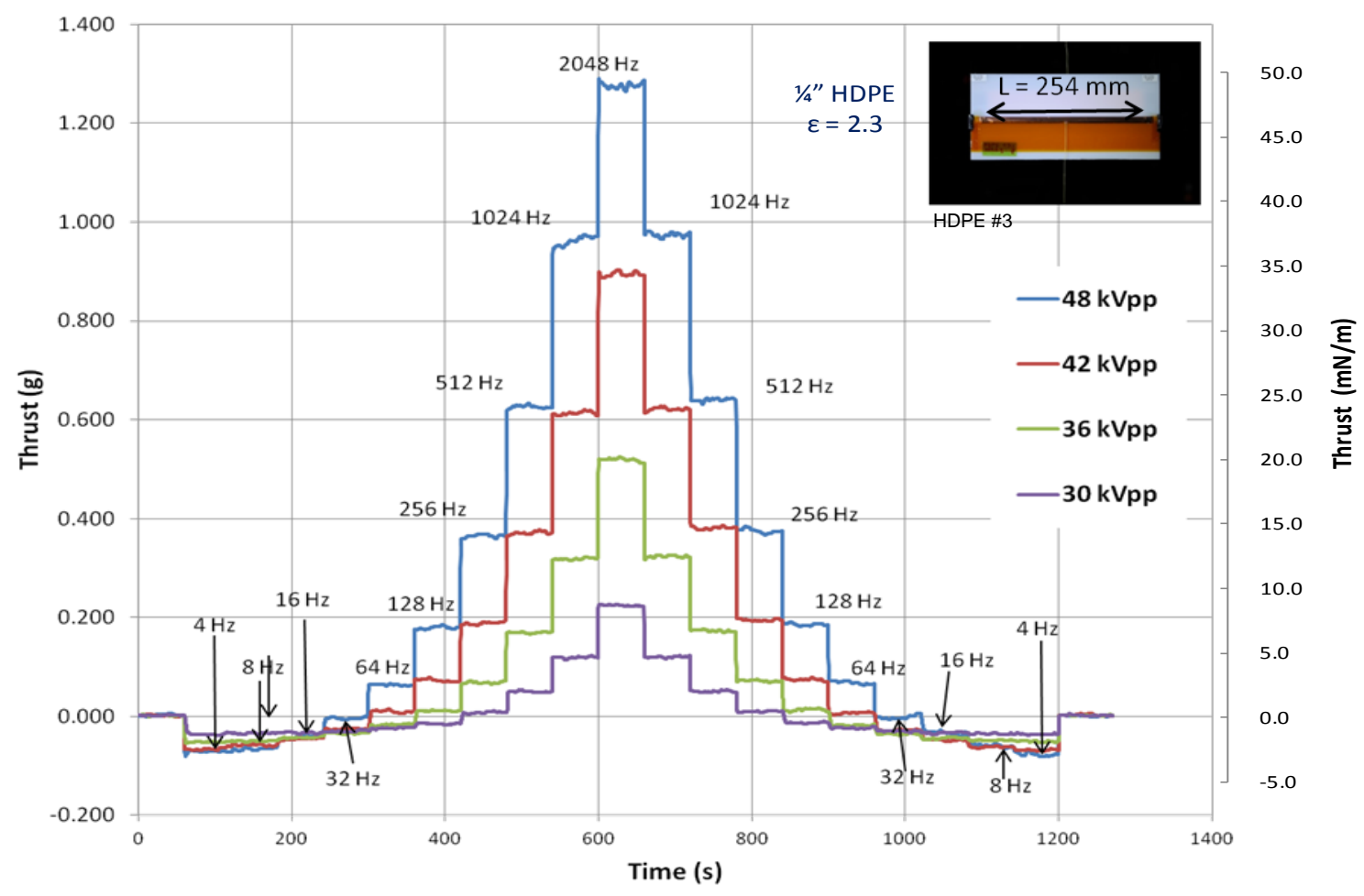

Figure 11. Frequency sweeps at constant voltages. RH 18\%, Dew Point $33{ }^{\circ}$ F. Sequence started at $48 \mathrm{kVpp} .1 \mathrm{~g}$ equals $38.6 \mathrm{mN} / \mathrm{m}$.

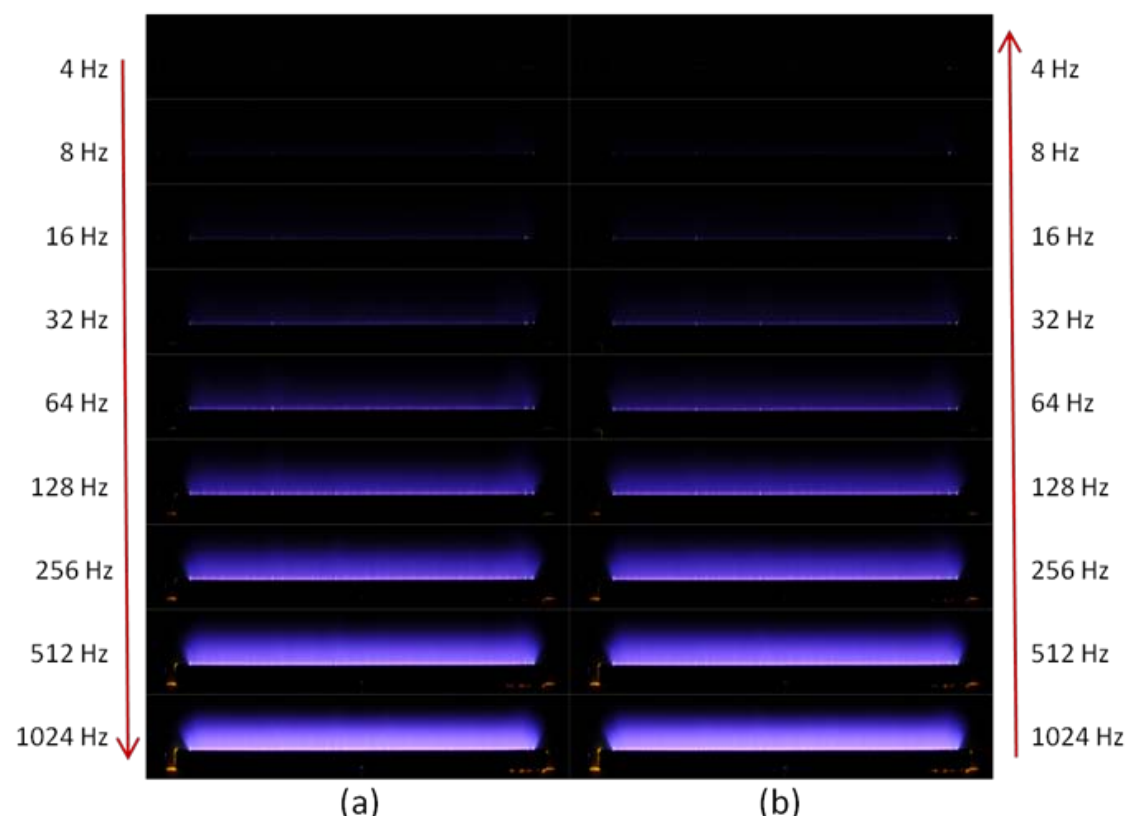

Figure 12. Images of $48 \mathrm{kVpp}$ frequency sweep discharges corresponding to Fig. 11 


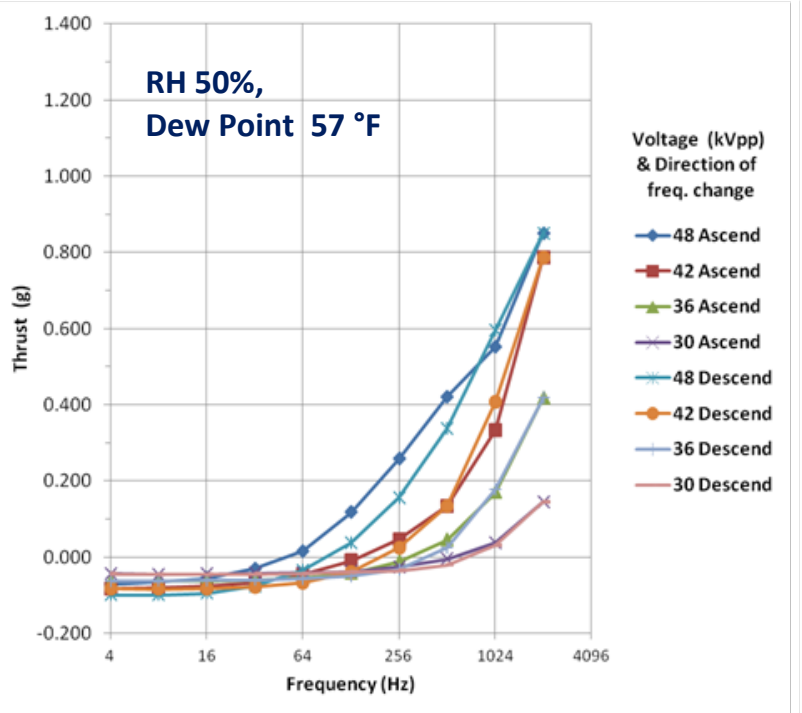

Figure 13. Total thrust as function of frequency at constant voltages. Humid case corresponding to Fig. 9. 1 g equals $38.6 \mathrm{mN} / \mathrm{m}$.

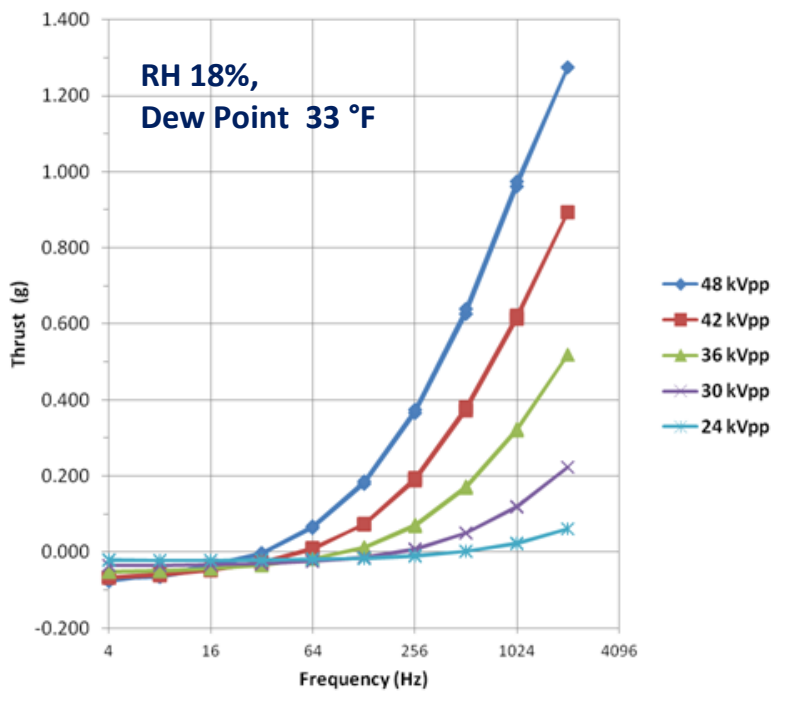

Figure 14. Total thrust as function of frequency at constant voltages. Low humidity case corresponding to Fig. 11. The ascending and descending parts overlap. $1 \mathrm{~g}$ equals $38.6 \mathrm{mN} / \mathrm{m}$.

The thrust data is extracted from the frequency sweeps by averaging the data within each 60 second step. We used a window smoothing function that generates a single averaged value of the thrust at each frequency step. The results are shown in Fig. 13 for the high humidity case. The low humidity case is shown in Fig. 14.

There are differences in the thrust level. The thrust is higher at the low humidity case. It is about $50 \%$ higher at $48 \mathrm{kVp}-\mathrm{p}$ and $2048 \mathrm{~Hz}$ in the drier ambient humidity.

In the drier case, the thrust corresponding to the ascending and descending frequencies curves virtually coincide. In the more humid case there are differences between the two. In particular, the curve at 48kVp-p is different during the ascending frequency curve than during the descending curve. The reason is that this particular ascending curve happened to be the first actuator energization after 4 days of rest. It served as unforced "burn-in" process. It experienced time-varying thrust changes above the typical baseline during this initial "burn-in" until it reached the highest frequency.

Even though we have not shown the standard deviation here, it is obvious that it is much larger in the humid case by examining the frequency sweeps steps (Figures 9 and 11).

\section{Anti-thrust}

When examining the results of the frequency sweeps shown above in Figures 9 and 11 we notice that the thrust is negative in a low frequency range between 4 and $32 \mathrm{~Hz}$ (even up to $64 \mathrm{~Hz}$ in other cases tested not shown here). To investigate further, we took detailed measurements of thrust vs. voltage at constant low frequencies, shown in Fig. 15. The test actuator used was HDPE \#2. The negative thrust values are noticeable. We examine the family of constant frequency curves and notice that they appear to coincide with the lowest frequency curve $(64 \mathrm{~Hz})$ at low voltages, then depart from that baseline with increasing voltage. The "departure" voltage for each curve appears to shift to the left to a lower voltage with each frequency increase. These observations motivated us to investigate if there is an ultimate lower frequency limit curve. We have repeated the test at constant frequency of $4 \mathrm{~Hz}$ for test actuator HDPE \#3, shown in Fig. 16. We were able to easily fit a parabolic curve to most of the points while acknowledging there are other points that depart from the natural parabola. Enhanced digital still images of 30 seconds exposure taken during the voltage sweep show that there is corona or plasma discharges at the voltage points that depart from the parabolic baseline, while no discharges are observed at the lower voltage points that fit well the same parabolic curve. In additional tests on other test articles we found that parabolic curve could always be 


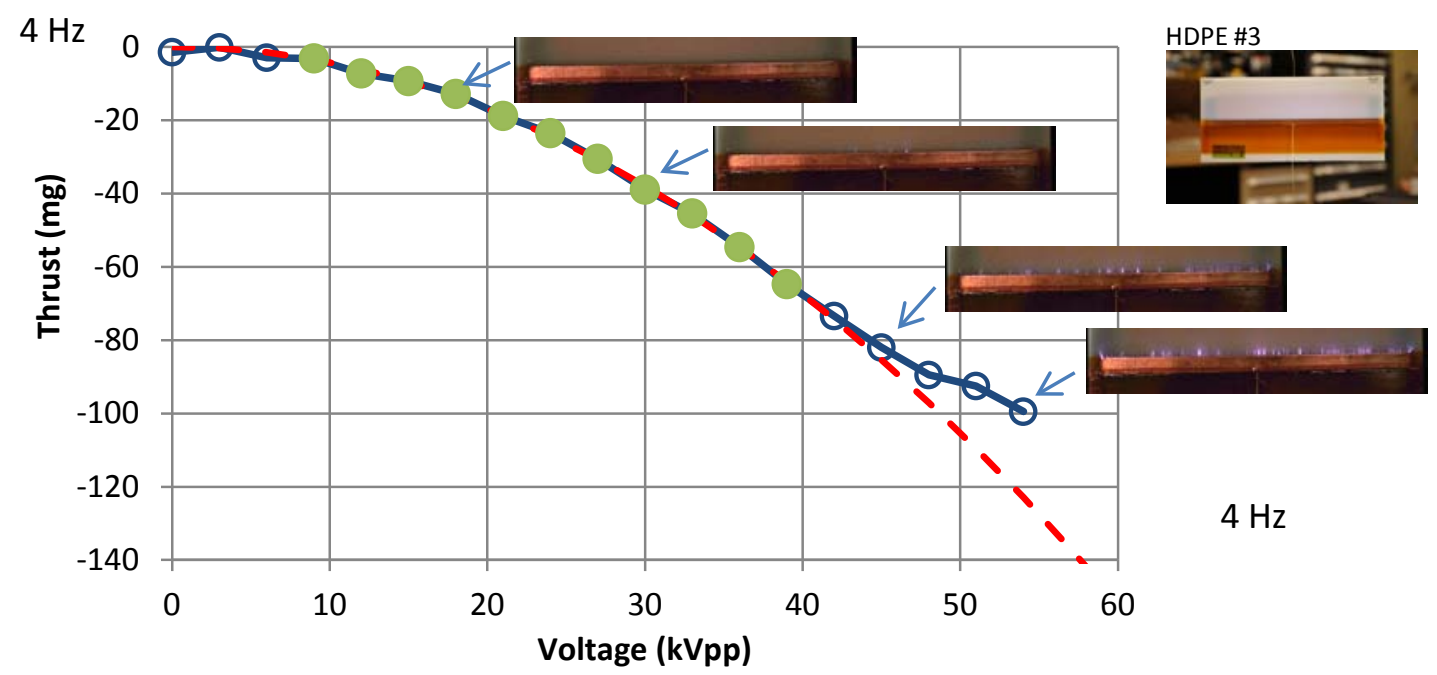

Figure 16. Thrust as function of voltage at constant frequency of $4 \mathrm{~Hz}$. Actuator HDPE \#3. Images are enhanced $30 \mathrm{~s}$ exposures. For this actuator $100 \mathrm{mg}$ equal $3.86 \mathrm{mN} / \mathrm{m}$.

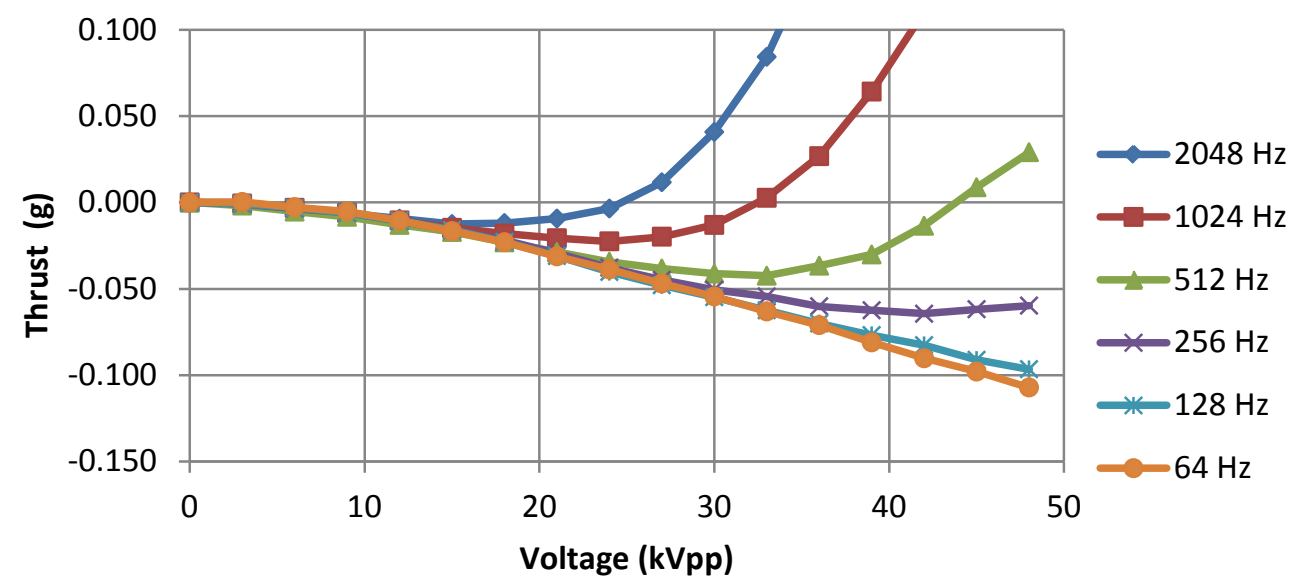

Figure 15. Thrust as function of voltage at constant frequencies. Actuator HDPE \#2. For this actuator $100 \mathrm{mg}$ equal $9.84 \mathrm{mN} / \mathrm{m}$.

fit to the low voltage range of averaged thrust values for low fixed frequencies when visible discharges were not present. The parabola was also found to be frequency independent.

These observations lead to formulate a hypothesis as follows,

$$
\text { Total Thrust }=\text { Plasma Thrust }+ \text { Anti Thrust }
$$

The total thrust is the thrust as measured by the balance. The plasma thrust is the thrust associated with the discharge on the exposed electrode that generates the momentum. The second term on the right hand side was named "anti-thrust" because it is always negative and is represented by the parabolic curve fit,

$$
\text { Anti Thrust }=k V^{2}
$$


We have found by performing additional tests under different conditions that the parabolic anti-thrust is confirmed to be frequency independent between 4 and $64 \mathrm{~Hz}$.

We make the following assumptions:

a) The anti-thrust is frequency independent at any frequency.

b) The anti-thrust is voltage dependent and can be extrapolated to higher voltages.

c) The anti-thrust is always present, including during visible plasma.

With these assumptions, the coefficient $k$ in Eq. 2 is a frequency- and voltage-independent constant. We therefore can use the anti-thrust parabola to separate the plasma thrust from the total thrust. The plasma thrust is the quantity of interest for characterizing the aerodynamic performance of the actuator. The anti-thrust curve, or the coefficient $k$ needs to be generated for each actuator and for each test installation by performing a voltage sweep at a low frequency (usually between 4 and $32 \mathrm{~Hz}$ in practice). It is very important to monitor voltage waveform as distortion will directly affect anti-thrust.

We propose that the anti-thrust always exists, but is masked by the thrust from visible plasma discharges as the voltage and frequency increase. We also propose that it is an installation-dependent effect that depends on the actuator surroundings and its own geometrical and dielectric properties. The $V^{2}$ dependence is similar to the voltage dependence of electrostatic forces. However, at this stage of the research, we have no experimental or computational verification to support a statement that the anti-thrust is caused by electrostatic forces. Further, the shear forces were assumed to be negligible in this discussion and are lumped in with the plasma thrust. Future research may be able to separate the shear force from the plasma force. We also have attempted to study the dependence of the anti-thrust on humidity, but we did not yet have sufficient data for conclusive results.

As an example, we performed the anti-thrust correction on the thrust data of the two cases shown earlier in Figures 13 and 14 and isolated the plasma thrust. The results are shown in Figures 17 and 18. Because a negative quantity was subtracted, the values of the plasma thrust increased relative to the measured thrust. This correction is substantial for smaller actuators and lower thrusts. We have found cases where the error can even exceed $100 \%$ if the correction is ignored.

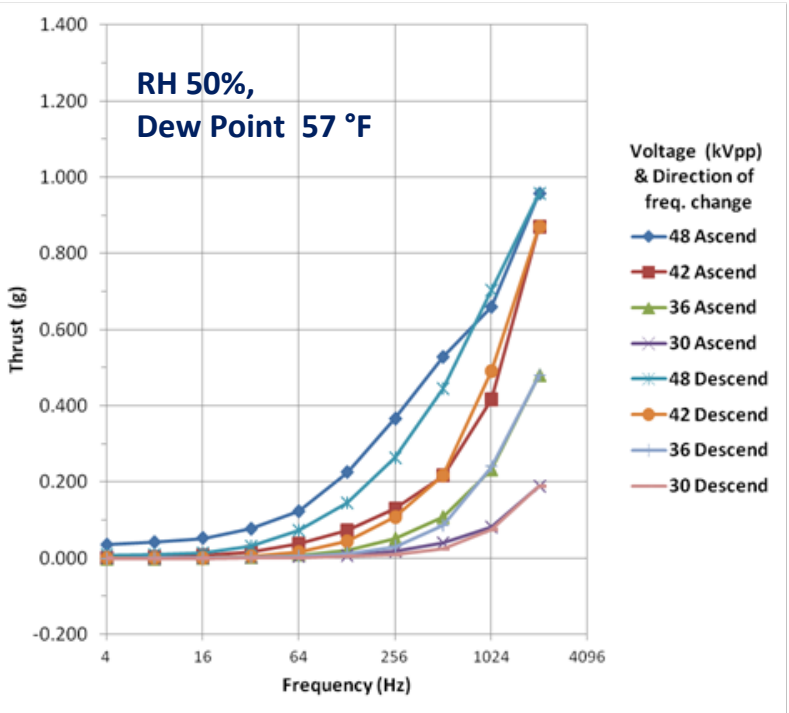

Figure 17. Isolated plasma thrust as function of frequency at constant voltages. Humid case.

Data of Fig. 13 after anti-thrust correction. 1 g equals $38.6 \mathrm{mN} / \mathrm{m}$.

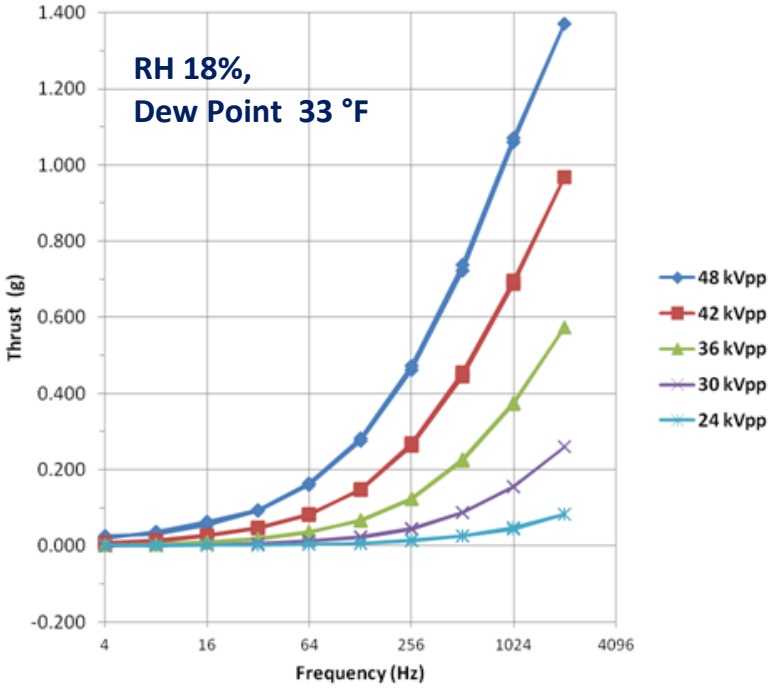

Figure 18. Isolated plasma thrust as function of frequency at constant voltages. Low humidity case. Data of Fig. 14 after anti-thrust correction. The ascending and descending parts overlap. 1 g equals $38.6 \mathrm{mN} / \mathrm{m}$. 


\section{Effect of the test installation}

To demonstrate the dependence on the test installation we performed a test with a smaller actuator, HDPE \#2 (Appendix A). The results are shown in Fig. 19. We first performed the anti-thrust measurement at $32 \mathrm{~Hz}$ on the freely suspended actuator. The results are shown in blue in Figure 19(a). The anti-thrust parabola was fitted and the deviation of the thrust from the parabola is noticeable starting at $30 \mathrm{kVp}-\mathrm{p}$.

We then placed a large-diameter, seamless, conductive cylinder around the actuator (304 stainless steel, 16 in. diameter, 59 in. long, 0.0375 in. thick [20 gauge], McMaster-Carr ${ }^{\circledR}$ part number 2538K652). The cylinder was suspended so the actuator was located inside it as shown in Fig. 19(c). The cylinder was grounded and the anti-thrust measurements repeated (plotted in green). There is marked difference between the unconfined actuator and the actuator installed within the grounded cylinder. The anti-thrust parabolic curves are different, the anti-thrust is larger with the grounded cylinder, and the thrust does not significantly deviate from the parabola. The measurements were repeated with the cylinder ungrounded, allowing its voltage potential to float. The results are plotted in red and are closer to the unconfined actuator case.

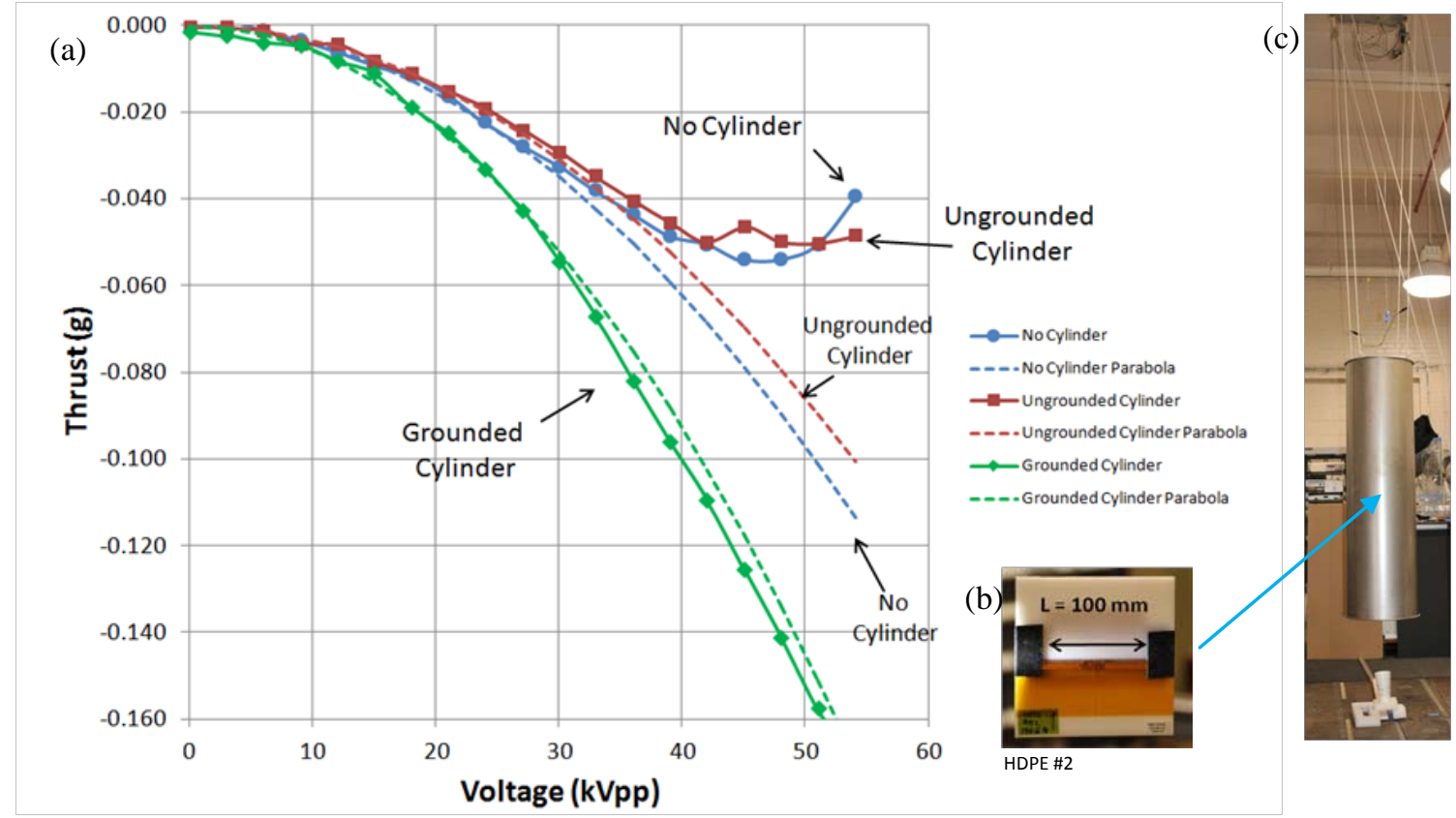

Figure 19. Anti thrust of an actuator with surrounding metal cylinder.

(a) Thrust as function of voltage at fixed frequency of $32 \mathrm{~Hz}$, dew point $60^{\circ} \mathrm{F}$, (b) Image of HDPE $6.3 \mathrm{~mm}$ thick actuator, (c) image of the suspended metal cylinder experimental setup. The actuator is suspended inside the cylinder at mid level as pointed by the arrow. 0.100 g equals $9.84 \mathrm{mN} / \mathrm{m}$.

These experiments serve as a significant validation of our anti-thrust hypothesis that implies that the anti-thrust is installation dependent. It seems that the surrounding material affected the electrical field around the actuator resulting in a different anti-thrust force.

Thrust measurements for the three cases are plotted in Fig. 20(a) as function of frequency for a constant voltage of $48 \mathrm{kVp}$-p. The negative thrust is observed at low frequencies. Above $1000 \mathrm{~Hz}$, the three cases appear to be overlapping or having small differences, and we may be lead to conclude that the actuator performance is identical within these three cases regardless of the surrounding cylinder. However, when the anti-thrust correction is performed to isolate the plasma thrust (results shown in Fig. 20[b]), it shows that the grounded cylinder case has larger plasma thrust compared to the open actuator case and that its performance is affected by the surrounding grounded sleeve. 


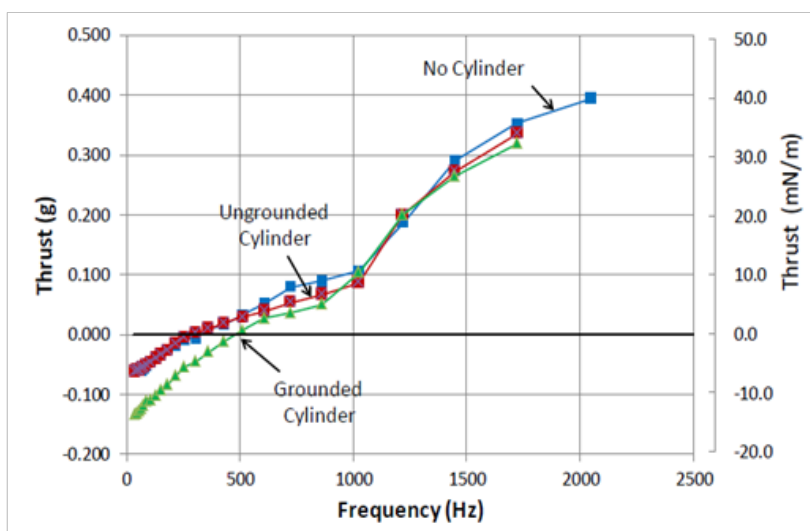

(a) Total thrust

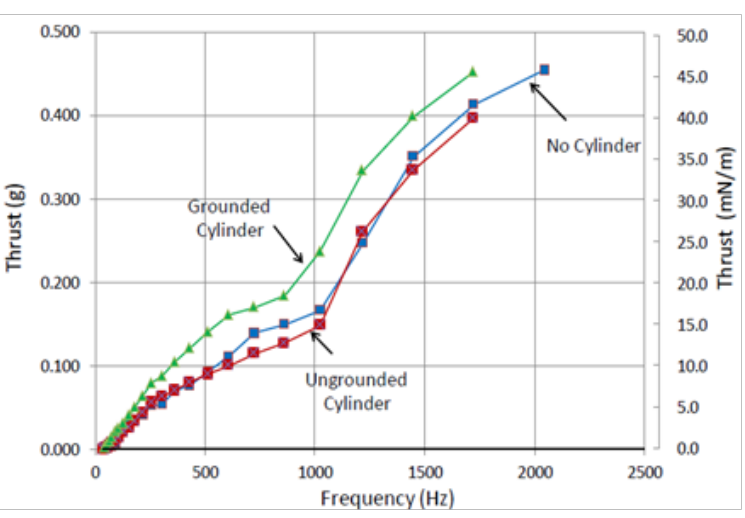

(b) Isolated plasma thrust

Figure 20. Thrust as function of frequency at constant voltage of $48 \mathrm{kVpp}$. (a) Total thrust, and, after anti-thrust correction, (b) Plasma thrust. $1 \mathrm{~g}$ equals $38.6 \mathrm{mN} / \mathrm{m}$.

\section{Conclusion}

The results shown were for sinusoidal waveforms in continuous-wave mode without pulsing or modulation. Some modifications and adaptations will be needed to evaluate other waveforms. This paper was not intended to provide comprehensive performance results of DBD actuators using thrust measurements, it was rather intended to expose a few considerations that need to be taken in studies of the aerodynamic performance of these devices. Our study includes: adopting a methodology to obtain consistent thrust results, defining an appropriate test setup, isolating the plasma force with our proposed anti-thrust hypothesis, and considering the effect of humidity. It is recommended to take extra care when comparing thrust performance of actuators between different setups and laboratories.

In addition to the effect of the issues discussed in this project, it needs to be noted that there is variability in construction of the test articles. Most of the studies reported in literature are performed with hand-made actuators. There can be problems with the insulation of sharp edges, the degree of variation in the fabrication, and the trapping of air bubbles within adhesives and between layers of dielectrics. The variability can accumulate to large error bars and make conclusions on relative performance of actuators prone to significant uncertainties.

In future work, we will investigate the source of the anti-thrust and attempt to confirm our hypothesis. It is most likely to be related to electrostatic forces between the actuator and the test installation. The electrostatic force field is a complex three-dimensional interaction that in principle can be calculated using numerical solution methods. Anticipated difficulties may be accurate calculation of electrostatic fields caused by sharp edges and corners and defining the electric potential and electric current of the surrounding objects.

The dependence on the installation indicates that the actuator performance in aerodynamic flow control application in a flight vehicle or a propulsion system will depend on its neighboring geometry, materials and electric potential. The surroundings will alter its performance. For example, if installed inside a jet engine, the actuator will be in very close proximity to grounded metallic surfaces; a situation different than when installed on an aircraft wing.

The inconsistent performance and the strong dependence on humidity has implications on the DBD actuator integration in a flow control system. If the actuator is installed as a component in an open control loop, there is less of a guarantee that the momentum it will provide will be as designed. A solution to this limitation may be inclusion of an active controller that will ensure it provides the desired momentum. Sufficient reserve power will be needed from the power supply. This problem does not exist when the actuator is incorporated in a closed control loop. The control loop will adjust the momentum it provides, but there still will be a requirement for sufficient reserve margin of the power supply. 


\section{Appendix A}

Actuators geometries and materials are as shown in Fig. 21. Table 1 lists dimensions and conversion factor to convert from thrust in grams (g) to normalized thrust (thrust per unit length of the exposed electrode) in millinewton per meter $(\mathrm{mN} / \mathrm{m})$.

The dielectric material, electrode material, and electrode insulation material used are also listed below.
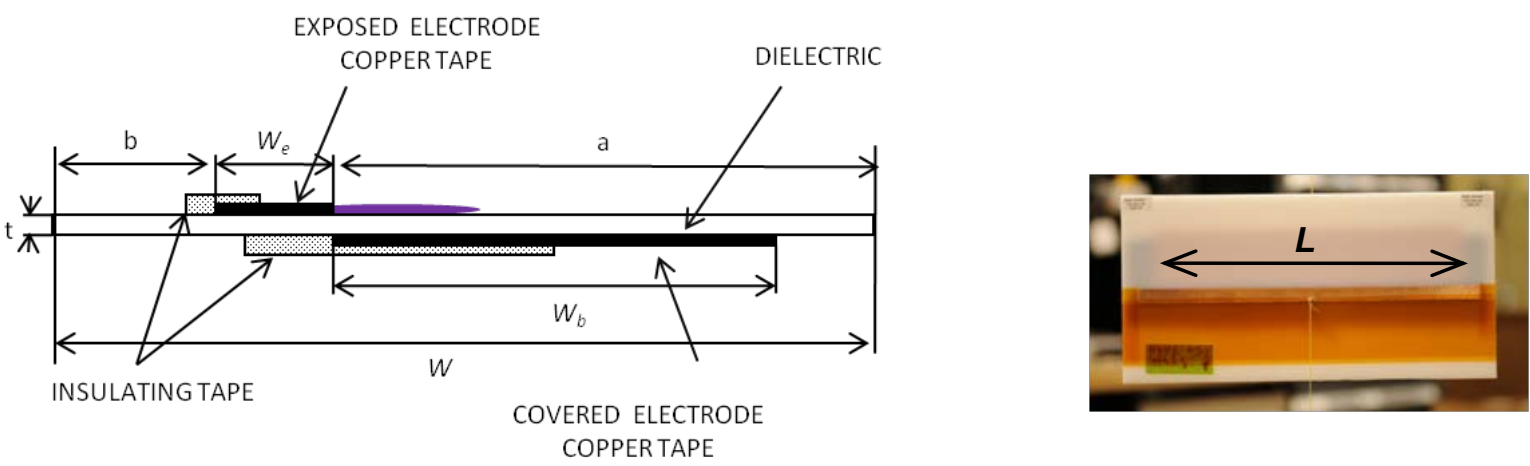

Figure 21. DBD plasma actuator test article - geometry and dimensions.

Table 1

Actuators Dimensions and information ${ }^{53}$

\begin{tabular}{|c|c|c|c|c|c|c|c|c|}
\hline Designation & $\boldsymbol{t}$ & $\boldsymbol{W}_{\boldsymbol{e}}$ & $\mathbf{W}_{\mathbf{b}}$ & $\mathbf{L}$ & $\mathbf{W}$ & $\mathbf{a}$ & $\mathbf{b}$ & $\begin{array}{c}\text { Thrust } \boldsymbol{T} \text { to } \boldsymbol{T} / \mathbf{L} \\
\text { Conversion Factor }\end{array}$ \\
\hline & $\mathrm{mm}$ & $\mathrm{mm}$ & $\mathrm{mm}$ & $\mathrm{Mm}$ & $\mathrm{mm}$ & $\mathrm{mm}$ & $\mathrm{mm}$ & $\mathrm{g}$ to $\mathrm{mN} / \mathrm{m}$ \\
\hline HDPE \#2 & 6.3 & 9.8 & 48.2 & 100 & 152 & 78 & 64.2 & 98.3616 \\
\hline HDPE \#3 & 6.3 & 9.8 & 49 & 254 & 151 & 75.5 & 65.1 & 38.6089 \\
\hline
\end{tabular}

a) Dielectric material used:

High Density Polyethylene (HDPE) . Nominal thickness $1 / 4$ in. McMaster-Carr ${ }^{\circledR}$ Inc. part number 8619K461.

HDPE Electrical properties ${ }^{6}$ :.

Dielectric Constant: 2.3 at $1 \mathrm{kHz}$

Dielectric Strength:: $22 \mathrm{MV} / \mathrm{m}$

Dissipation Factor: 0.0005 at $1 \mathrm{kHz}$

b) Electrodes material:

Copper tape with conductive adhesive. $3 \mathrm{M}^{\circledR}$ No. 1181

Copper thickness: $0.04 \mathrm{~mm}(1.4 \mathrm{mil})$

Adhesive thickness: $0.03 \mathrm{~mm}(1.2 \mathrm{mil})$

c) Electrode insulation materials:

Covered electrode: $3 \mathrm{M}^{\circledR}$ Scotch-Seal ${ }^{\mathrm{TM}}$ No. 229 pads

Exposed electrode::

Kapton ${ }^{\circledR} 3 \mathrm{M}{ }^{\circledR}$ No. 5413, $0.08 \mathrm{~mm}$ (3 mil) thick

Super Corona Dope: MG Chemicals ${ }^{\circledR}$ Inc. Cat. No. 4226-1L

${ }^{5}$ Due do fabrication inaccuracies the sum $\mathrm{a}+\mathrm{b}+W_{\mathrm{e}}$ does not equal $\mathrm{W}$

${ }^{6} \mathrm{http}: / /$ www.azom.com as of December 12, 2013

American Institute of Aeronautics and Astronautics 


\section{Acknowledgments}

This project was supported by the National Aeronautics and Space Administration (NASA) Aerospace Sciences Project of the Fundamental Aeronautics Program.

The Schlieren image was generated with the help of Dr. Amy F. Fagan of NASA Glenn Research Center. We thank Prof. Annie Leroy-Chesneau for permission to reprint Fig. 5.

\section{References}

\footnotetext{
${ }^{1}$ Moreau, E., “Airflow Control by Non-Thermal Plasma actuators,” Journal of Physics. D: Applied Physics, Vol. 40, 2007, pp. 605-636.

${ }^{2}$ Corke, T. C., Post, M. L., and Orlov, D. M., “SDBD Plasma Enhanced Aerodynamics: Concepts, Optimization and Applications,” Progress in Aerospace Sciences Vol. 43, 2007, pp. 193-217.

${ }^{3}$ Corke, T. C., Post, M. L., and Orlov, D. M., "Single Dielectric Barrier Discharge Plasma Enhanced Aerodynamics: Physics, Modeling and Applications,” Experiments in Fluids, Vol. 46, 2009, pp. 1-26.

${ }^{4}$ Corke, T. C., Enloe, C. L., and Wilkinson, S. P., "Dielectric Barrier Discharge Plasma Actuators for Flow Control,” Annual Reviews of Fluid Mechanics, Vol. 42, 2010, pp. 505-29.

${ }^{5}$ Roupassov, D. V., Nikipelov, A. A, Nudnova, M. M., and Starikovskii, A. Yu., "Flow Separation Control by Plasma Actuator with Nanosecond Pulse Periodic Discharge,” AIAA Paper 2008-1367, 2008

${ }^{6}$ Glezer, A., and Amitay, M., “Synthetic Jets,” Annual Reviews of Fluid Mechanics, Vol. 34, 2002, pp.:503-29.

${ }^{7}$ Hoskinson, A .R., Hershkowitz, N., and Ashpis, D. E., "Force Measurements of Single and Double Barrier DBD Plasma Actuators in Quiescent Air,” Journal of Physics D: Applied Physics, Vol. 41, 2008, 245209 (9pp).

${ }^{8}$ Baughn, J. W., Porter, C. O,, Peterson, B. L., McLaughlin, T. E., Enloe, C. L., Font, G. I., and Baird, C., ”Momentum Transfer for an Aerodynamic Plasma Actuator with an Imposed Boundary Layer, “AIAA paper 2006-168, 2006.

${ }^{9}$ Durscher, R., and Roy, S., "Evaluation of Thrust Measurement Techniques for Dielectric Barrier Discharge Actuators," Experiments in Fluids Vol. 53, 2012, pp. 1165-1176.

${ }^{10}$ Opaits, D. F., et al, “Surface Plasma Induced Wall jets,” AIAA Paper 2010-469, 2010

${ }^{11}$ Glauert M. B., “Wall Jet,” Journal of Fluid Mechanics, Vol. 1, 1956.

${ }^{12}$ Boucinha, V., Magnier, P., Leroy-Chesneau, A., Weber, R., Joussot, R., Dong, B., Hong, D., “Characterization of the Ionic Wind Induced by a Sine DBD Actuator used for Laminar-to-Turbulent Transition Delay,” AIAA Paper 2008-4210, 2008.

${ }^{13}$ Ashpis, D. E., Laun M. T., and Griebeler, E. L., "Progress toward Accurate Measurements of Power Consumptions of DBD Plasma Actuators,” AIAA Paper 2012-0823, 2012.

${ }^{14}$ Koo, I. G., Cho, J. H., Lee, W. M., "Influence of Gas Humidity on the Uniformity of RF-Powered Atmospheric-Pressure Low-Temperature DBD Plasmas”, Plasma Processes and Polymers, Vol. 5, 2008, pp. 161-167.

${ }^{15}$ Farouk, T., Farouk, B., Gutsol, A., and Fridman, A., “Atmospheric Pressure Radio Frequency Glow Discharges in Argon: Effects of External Matching Circuit Parameters”, Plasma Sources Science and Technology, Vol. 17, 2008, 035015 (15pp).
} 